FSS

Luo, J., Li, H., Yeung, P., \& Chang, C. (2021). The association between media multitasking and executive function in Chinese adolescents: Evidence from self-reported, behavioral and fNIRS data. Cyberpsychology: Journal of Psychosocial Research on Cyberspace, 15(2), Article 8. https://doi.org/10.5817/CP2021-2-8

\title{
The Association Between Media Multitasking and Executive Function in Chinese Adolescents: Evidence From Self-reported, Behavioral and fNIRS Data
}

\author{
Jiutong Luo ${ }^{1,2,3}$, Hui Li ${ }^{4}$, Pui-sze Yeung ${ }^{3}$, \& Chunqi Chang $5,6,7$ \\ ${ }^{1}$ Advanced Innovation Center for Future Education, Faculty of Education, Beijing Normal University, Beijing, China \\ ${ }^{2}$ Center for Educational Science and Technology, Beijing Normal University at Zhuhai, Guangdong Zhuhai, China \\ ${ }^{3}$ Faculty of Education, The University of Hong Kong, HKSAR, China \\ ${ }^{4}$ School of Education, Macquarie University, Sydney, Australia \\ ${ }^{5}$ School of Biomedical Engineering, Health Science Center, Shenzhen University, Shenzhen, China \\ ${ }^{6}$ Guangdong Provincial Key Laboratory of Biomedical Measurements and Ultrasound Imaging, Shenzhen University, \\ Shenzhen, China \\ ${ }^{7}$ Center for Neuroimaging, Shenzhen Institute of Neuroscience, Shenzhen, China
}

\begin{abstract}
This study examined the association between media multitasking and executive function in Chinese adolescents by comparing heavy/high and light/low media multitaskers, i.e., HMMs and LMMs, with self-reports, behavioral measures and functional near-infrared spectroscopy (fNIRS). The participants were 12 HMMs (media multitasking scores above the 75th percentile) and $10 \mathrm{LMMs}$ (media multitasking scores below the 25th percentile) chosen from a sample of 61 adolescents. Each participant completed a self-reported questionnaire on executive function and three executive function cognitive tasks: 2-back, Color Stroop, and Number-letter Determination) while wearing the fNIRS. The results indicated that: (1) the HMMs showed more impairment in executive function than the LMMs based on questionnaire data analysis; (2) there were no significant differences between the HMMs and LMMs in their performance on the cognitive tasks; and (3) the HMMs showed greater prefrontal activation than the LMMs during the 2-back and Color Stroop tasks. These findings implied that media multitasking might be associated with the reduced effectiveness in the brain areas responsible for executive function. These findings provide evidence of the negative relationship between media multitasking and executive function; and indicated the benefits of using multiple assessment methods in studying this topic.
\end{abstract}

Keywords: Media multitasking; executive function; multiple perspectives; fNIRS; heavy/light media multitaskers

\section{Introduction}

Media multitasking, an increasingly prevalent phenomenon among adolescents, has attracted growing research interest from cognitive scientists (Foehr, 2006; Ophir et al., 2009; Wallis, 2010). Many scholars have sought to understand the negative impact of media multitasking on cognitive functioning by comparing heavy/high media multitaskers (HMMs, i.e., mean +1 standard deviation [SD] or upper quartiles) and light/low media multitaskers (LMMs, i.e., mean - 1 SD or lower quartiles; e.g., Ophir et al., 2009; Sanbonmatsu et al., 2013). Moreover, in comparison to LMMs, HMMs perform worse on attention, working memory, and task-switching tasks (Baumgartner et al., 2018; Cardoso-Leite et al., 2016; Ophir et al., 2009; Wiradhany \& Nieuwenstein, 2017); score 
higher on psychological factors, such as sensation-seeking and impulsiveness; and report lower on self-esteem (Luo, Yeung, et al., 2020a, 2020b) and well-being (e.g., Becker et al., 2013; Sanbonmatsu et al., 2013). Recently, studies have also investigated the remedial interventions (e.g., awareness, restriction, and mindfulness interventions) for media multitaskers, and have mainly targeted improving their executive function performance (see Parry \& le Roux, 2019, for a review). To date, the findings on the relationship between executive function and media multitasking experience are still mixed (for a review, see Uncapher et al., 2017). Most of the existing studies have compared the behavioral performance of HMMs and LMMs using cognitive behavioral measures, and a limited number of studies have examined the issue of media multitasking by analyzing neuroimaging data (Loh \& Kanai, 2014; Moisala et al., 2016). Little is known about the correspondence between the behavioral and neuroimaging findings. To fill this literature gap, this study adopted a transdisciplinary approach (using selfreported, behavioral, and neuroimaging evidence) to study the association between media multitasking and executive function in Chinese adolescents.

\section{Conventional Versus Neuroimaging Approaches to Examining Executive Function}

Executive function (EF) refers to the set of cognitive processes that are essential to goal-directed, efficient, and adaptive behavior (Huizinga et al., 2006; Huizinga \& Smidts, 2010). Previous research using latent variable analysis has identified three core EF processes: working memory, inhibition, and shifting (Miyake et al., 2000). Besides, research has also revealed that the development of EF is a protracted process, that continues into early adulthood (Diamond, 2013; Huizinga \& Smidts, 2010). During the adolescent transition, the regulatory systems gradually come under the control of executive function (Steinberg, 2005); therefore, adolescents may be more sensitive to the long-term effects that could affect their executive function development. In general, EF has been shown to be strongly related to the brain's prefrontal cortex and has been extensively explored by cognitive scientists using various approaches (Huizinga et al., 2006). The first and foremost is the conventional approach, which assesses an individual's executive function through self-reported questionnaires and cognitive tasks. Several questionnaires, such as the Dysexecutive Questionnaire (DEX) and Behavior Rating Inventory of Executive Function (BRIEF), have been developed and widely used to examine executive function (Burgess et al., 1998; Gioia et al., 2015; Wilson et al., 1998). Several cognitive task paradigms, such as the n-back, digit span, the Stroop task, and the Wisconsin Card Sorting Task (WCST), are also well established measures of various aspects of executive function (Diamond, 2013). High scores on the DEX and BRIEF surveys indicate low executive function, whereas for the cognitive tasks, low executive function manifests as weak behavioral performance (e.g., low accuracy, long response times).

Recently, the neuroimaging approach has been increasingly used to explore the neural correlates of executive function. Studies using functional magnetic resonance imaging (fMRI), functional near-infrared spectroscopy (fNIRS) and magnetoencephalography (MEG) have reported that executive function involves a wide range of brain areas, especially the prefrontal cortex (PFC; e.g., Moriguchi \& Hiraki, 2013; Parker, 2012; Zhao et al., 2016). In particular, it has been found that both the anterior dorsolateral prefrontal cortex (DLPFC) and the anterior ventrolateral prefrontal cortex (VLPFC) are involved in inhibition ability (Laguë-Beauvais et al., 2013). Other studies have indicated that working memory involves the DLPFC and the left frontal opercula areas (León-Domínguez et al., 2015). In most cases, increased brain activation in these areas, especially the prefrontal area, is related to a better executive function task performance (Zhao et al., 2016).

The conventional and neuroimaging approaches are not in conflict; rather, they can complement and confirm each other. However, although research on executive function has advanced in the past decades, very few studies have adopted a transdisciplinary approach to compare the evidence from self-reported, behavioral, and neuroimaging approaches to confirm the association between media multitasking and executive function. For instance, fNIRS is a non-invasive neuroimaging technique that can measure changes in the levels of oxygenated hemoglobin $(\mathrm{HbO})$ and deoxygenated hemoglobin $(\mathrm{HbR})$ in the brain, especially surficial areas, thus revealing the activation and involvement of the brain areas involved in a specific task (Strangman et al., 2002). Adolescents' executive functioning is highly correlated with the development of the prefrontal cortex (Dumontheil, 2016), which can be measured using fNIRS. Therefore, this study aimed to fill the methodological gap in media multitasking and executive function research using this neuroimaging method. 


\section{Self-Reported, Behavioral, and Neuroimaging Approaches to Media Multitasking Research}

Media multitasking refers to the simultaneous engagement in multiple media tasks (Foehr, 2006; Ophir et al., 2009; Wallis, 2010) and has been explored with various cognitive approaches. Some studies using conventional approaches such as self-reports have found that media multitasking is associated with low self-control and poor executive function (Luo \& Liang, 2018; Magen, 2017; Sanbonmatsu et al., 2013; Shin et al., 2019). This evidence was mostly based on impulsivity and sensation-seeking measures and might be inconsistent with those no correlation results (Shih, 2013). Another study, which used the Behavior Rating Inventory of Executive Function (BRIEF) to measure executive function among media multitaskers (Baumgartner et al., 2014), found that all three of the studied cognitive dimensions (i.e., working memory, inhibition, and shifting) were all correlated with media multitasking. However, the study did not provide empirical evidence of any relationship between media multitasking and general executive function measures, such as the Dysexecutive Questionnaire (DEX). It also did not compare the differences between HMMs and LMMs on these measures. Therefore, further investigations using the self-reported method are still needed in this aspect. Accordingly, this study hypothesized that HMMs would report more problems using the self-reported measures of executive function (Hypothesis 1).

Another widely used approach to explore the relationship between media multitasking and executive function is the behavioral approach, which is based on cognitive tasks. The existing results have generally supported a negative association between media multitasking and executive function, including working memory (Cain et al., 2016; Cardoso-Leite et al., 2016), inhibition (Murphy \& Creux, 2021), and shifting (Ophir et al., 2009; Wiradhany \& Nieuwenstein, 2017). However, some studies have failed to replicate these findings and have even reported conflicting results as well (see Wiradhany \& Nieuwenstein, 2017, for a review). Within the literature, the pattern of evidence is less clear for associations with working memory and shifting aspects than the inhibition aspect. For example, Baumgartner et al. (2014) did not find any difference in shifting (i.e., switch-cost) between HMMs and LMMs. In addition, Minear et al. (2013) found no differences in working memory between these groups on a reading span task. Regarding the inhibition, however, the significant marginal effect also requires further replication studies, and more investigations of the behavioral performance of media multitaskers on cognitive tasks are needed. According to individual studies included in the meta-analysis conducted by Wiradhany and Nieuwenstein (2017), the negative associations between media multitasking and executive function task performances are most common. To this end, we hypothesized that HMMs would show poorer behavioral performance than LMMs in executive function tasks (Hypothesis 2).

Recently, the neuroimaging approach has been employed to complement and confirm the findings of behavioral performance studies. For instance, an fNIRS study on media multitasking in adolescents and young adults found that HMMs showed higher brain activation in prefrontal regions than LMMs in a sentence comprehension task with distraction (Moisala et al., 2016). This finding indicated that HMMs might need extra "recruitment of brain areas involved in attentional and inhibitory control" (Moisala et al. 2016, p. 113). Another study using structural imaging technique, showed that HMMs had lower gray-matter density in the anterior cingulate cortex than LMMs (Loh \& Kanai, 2014). These two studies implicitly revealed the important role of prefrontal areas in media multitasking. Given the fact that executive function is associated with the dorsolateral prefrontal cortex (DLPFC or BA9 and BA46; e.g., Koike et al., 2013; Laguë-Beauvais et al., 2013; León-Domínguez et al., 2015; Zhao et al., 2016), and that the multitasking ability is more associated with the anterior prefrontal cortex (APFC or BA10; e.g., Dreher et al., 2008; Loh \& Kanai, 2014; Roca et al., 2011), we hypothesized that media multitasking would have a negative relationship with the function of prefrontal brain areas. This study further tested this hypothesis via neuroimaging evidence. More specifically, according to the higher activation among HMMs than LMMs (Moisala et al., 2016), this study also hypothesized that HMMs would have greater brain activation than LMMs during cognitive tasks (Hypothesis 3).

\section{The Present Study}

The above literature review has indicated that the existing findings from both self-reports and behavioral performance studies are mixed and even conflicting, and thus might not be adequate to reveal the association between media multitasking and executive function. Therefore, we believe that neuroimaging evidence of the differences between HMMs and LMMs in executive function tasks should provide more robust evidence of the association between media multitasking and the user's brain activity. In this case, this study aimed to provide 
triangulated data from a psychological questionnaire, cognitive task and neuroimaging to systematically explore the differences in executive function between HMMs and LMMs.

\section{Method}

\section{Participants}

Altogether 22 adolescents (aged between 14-17 years old; $M_{\text {age }}=16.05, S D=0.84 ; 7$ boys and 15 girls) participated in this study. They were recruited through approaching their schools and teachers and snowball sampling via their peers. In total, 61 adolescents initially consented to participate in this study. The average media multitasking score was 2.79 (SD = 0.66). Then, they were divided into two groups (i.e., HMMs and LMMs) based on their scores on the media multitasking scale (MMS), which reflected their media multitasking performance. Although the sample size was relatively smaller, they were recruited with very similar age groups from the same schools. In comparison to other studies that have used the same MMS (Luo, Yeung, et al., 2020a, 2020b), the average media multitasking score in this study $(M=2.79)$ was slightly higher than the previously reported two studies $(M s=2.25-2.32)$. We might suspect that adolescents' other indicators, such as gender, personality, ICTs usage, etc. (Chen et al., 2021), affected this result.

Among the 61 participants, twelve scored higher than upper quartiles were included in the HMMs group, and ten scored less than lower quartiles and thus were included in the LMMs group. The other 39 scored around the mean, including those who were right on the upper or lower quartiles, and were excluded from the study because the primary aim was to compare HMMs and LMMs. All 22 of the included participants had a normal or corrected-tonormal vision and were right-handed. Chi-square tests revealed no significant gender $(p=.452)$ or age $(p=.474)$ differences between the two groups (see Table 1). The research was approved by the Human Research Ethics Community from the University of Hong Kong. All of the adolescents' parents provided their informed consent, and all of the adolescents completed a written consent form before participating in the study.

Table 1. Demographic Information of the LMMs and HMMs Groups.

\begin{tabular}{lcccc}
\hline & LMMs $(n=10)$ & HMMs $(n=12)$ & LR/t & $p$ \\
\hline Gender (M:F) & $4: 6$ & $3: 9$ & .565 & .452 \\
Age (SD) & $15.90(1.10)$ & $16.17(.58)$ & -.73 & .474 \\
\hline Note. M = Male; F = Female; LMMs = Light media multitaskers; HMMs = Heavy media multitaskers. The \\
statistical value is the likelihood ratio (LR) test result (expected value less than 5) for gender or t-test for age.
\end{tabular}

\section{Measures}

\section{Media Multitasking Scale (MMS)}

The MMS is a 14-item scale developed for Chinese adolescents (Luo et al., 2018). It contains three constructs, i.e., multitasking across two media (MAM; five items; e.g., "while watching TV/video, I check or send (voice) messages"), multitasking with media and non-media (MMNM; four items; e.g., "while eating, I watch TV/video"), and concentration without multitasking (CWM; reverse-coded, five items; e.g., "I can focus on talking to one person on the phone/video call without doing other things"). All items were measured on a five-point Likert scale ( $1=$ "never", $5=$ "always"). The participants' scores indicate their level of media multitasking in daily life. And the included participants were grouped into HMMs (12 participants; upper quartiles) and LMMs (10 participants; lower quartiles), accordingly. An independent study used unidimensional MMS with second-order confirmatory factor analysis and found it to have satisfactory reliability and good convergent validity (Luo et al., 2018). The Cronbach's a was .95 in the present study.

\section{Executive Function Measures}

Two self-reported measures were used to evaluate the participants' executive functioning. The first measure was adapted from the Behavior Rating Inventory of Executive Function (Second Edition, BRIEF 2) to measure three dimensions of executive function (Gioia et al., 2015): working memory (e.g., "when I am given three things to do, I remember only the first or last"), inhibition (e.g., "I have trouble sitting still"), and shifting (e.g., "I have trouble 
accepting a different way to solve a problem with things such as schoolwork, friends, or tasks"). Each dimension contains eight items measured on a 3-point Likert scale ( $1=$ "never", $3=$ "often"). The participants who scored higher on each dimension were identified as more "problematic" in the relevant aspect of their daily lives. The internal consistency for three dimensions (i.e., working memory, inhibition, and shifting) in this study was .85, .77, and .82, respectively. The second measure was the 20-item Dysexecutive Questionnaire (DEX; Burgess et al., 1998; Wilson et al., 1998), which was used to evaluate the participants' general executive function in general (e.g., "I have problems in understanding what other people mean unless they keep things simple and straightforward"). Each item was measured on a 5-point Likert scale ( 1 = "strongly disagree", $5=$ "strongly agree"). A higher score on this scale indicated a higher frequency of dysexecutive behavior in daily life. The Chinese version was shown to be reliable and valid in Chan's (2001) study, and its internal consistency was .89 in this study.

\section{N-Back Task}

The participants were asked to finish three blocks of the n-back (in this study, the 2-back) task. This task was commonly used in previous media multitasking studies (see Wiradhany \& Nieuwenstein, 2017, for a review). Each block included 15 trials, resulting in a time of approximately 30s for each block. Although the previous literature usually used more trials in their behavioral experiments, this study chooses to adopt 15 trials, i.e., around 30s for each block, a typical fNIRS block design to record blood signal (Basura et al., 2018; Herold et al., 2018). This block design was also applied in the Color Stroop and number-letter tasks (see below). In the 2-back task, letters were presented to the participants for $500 \mathrm{~ms}$, followed by a fixation point (+) in the center of the screen for $1500 \mathrm{~ms}$ (see Figure 1a). The participants were instructed to press " 1 " if the presented letter was the same as the letter presented two letters earlier and to press " 2 " if it was not. The correct letters (or targets) were varied ( $6 \pm 1$ targets) in each block. The participants were also given time to practice several trials before starting. This task has been widely used to assess working memory in previous studies (León-Domínguez et al., 2015).

\section{Color Stroop Task}

The participants were presented with words ("red," "yellow," "blue," and "green") in different colors. In each trial, these words were randomly inked in different colors (i.e., red, yellow, blue, and green, respectively; see Figure 1b). This task was chosen because it places relatively low demand on other aspects of executive function aspects, such as working memory. A similar task paradigm has also been used in the media multitasking research (Murphy \& Creux, 2021). The participants were instructed to indicate the inked color rather than the meaning of the word by pressing the corresponding numbers, as they had trained in the practice session. This task also consisted of 3 blocks, with 15 trials in each block. Each word was shown for $1500 \mathrm{~ms}$, with a fixation point (+) shown for $500 \mathrm{~ms}$ afterwards. This task has been widely used to measure inhibition in previous studies (e.g., Schroeter et al., 2004).

\section{Number-Letter Task}

The participants were asked to determine whether the presented number was an odd (i.e., 3, 5, 7, 9) or even (i.e., $4,6,8,0$ ), or whether the shown letter was a consonant (i.e., P, K, N, S) or vowel (i.e., A, E, I, U) (see Figure 1c). The letter "O" was not included to prevent confusion with " 0 " (zero), used in the numbers. This task was used in the pioneering research on media multitasking and executive function (Ophir et al., 2009). The participants were asked to practice a few trials before starting. There were also three blocks in this session, with 15 trials in each block. In this task, the participants were shown either "number" or "letter" for $200 \mathrm{~ms}$ to tell them which they should focus on in the next screen. A randomly paired number and letter were then displayed to them for $1500 \mathrm{~ms}$ and followed by a $300 \mathrm{~ms}$ interval. The participants needed to decide according to the above rules and then pressed the corresponding key (for more details, see Ophir et al., 2009). This task has been used to measure shifting ability in previous studies (Ophir et al., 2009). 
Figure 1. Paradigm for 2-Back (a), Color Stroop (b) and Number-Letter (c) Tasks.

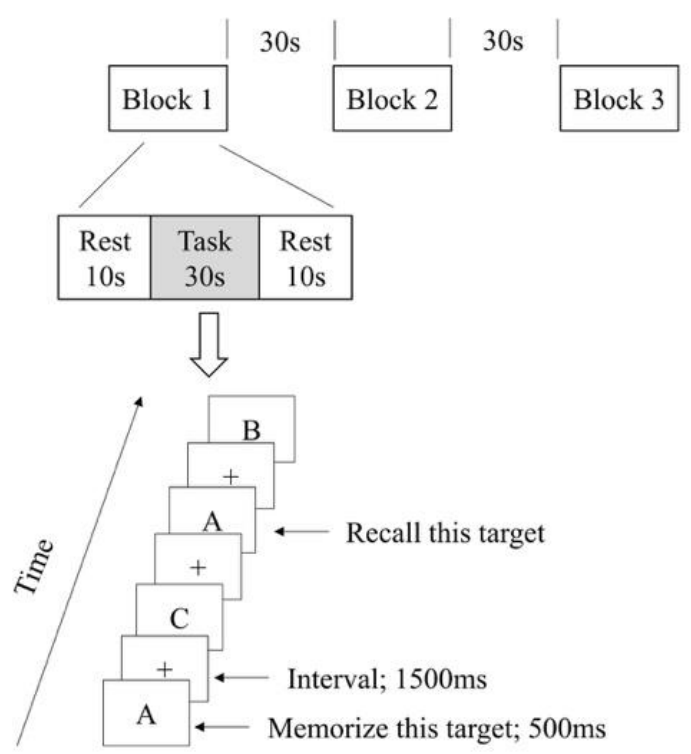

(a) 2-back

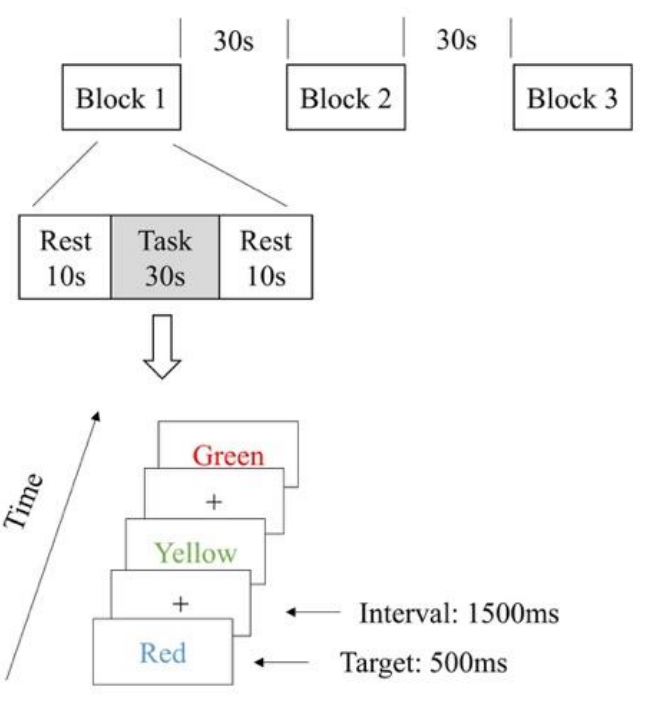

(b) Color Stroop
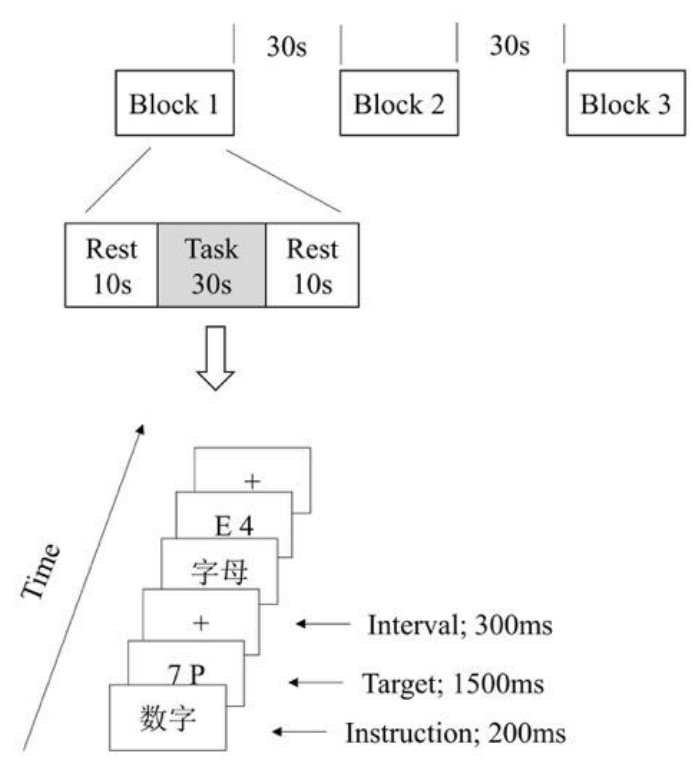

(c) Number-letter

\section{Functional Near-Infrared Spectroscopy (fNIRS)}

A 20-channel fNIRS system (Oxymon Mk III, Artinis, The Netherlands) was applied to simultaneously measure the concentration changes of oxygenated hemoglobin $(\mathrm{HbO})$ and deoxygenated hemoglobin $(\mathrm{HbR})$ in the participants' brains. This system employs two different wavelengths in the near-infrared range (i.e., $760 \mathrm{~nm}$ and $850 \mathrm{~nm}$ ) to measure the changes in optical density and then converts the data into changes in the concentrations of $\mathrm{HbO}$ and $\mathrm{HbR}$ using the modified Beer-Lambert law. The channels were located following the international 10-20 system for EEG, with a $3 \mathrm{~cm}$ distance between each paired emitters and detectors. According to A. Ducan et al. (1996), the subject-specific differential path-length factor (DPF) constant was calculated based on each participant's age before starting the experiment. For all participants, the channels covered their DLPFC (BA9 and BA 46) and APFC (BA10). The details of the channel placement are shown in Figure 2. The sampling rate for data acquisition was 50 $\mathrm{Hz}$. 

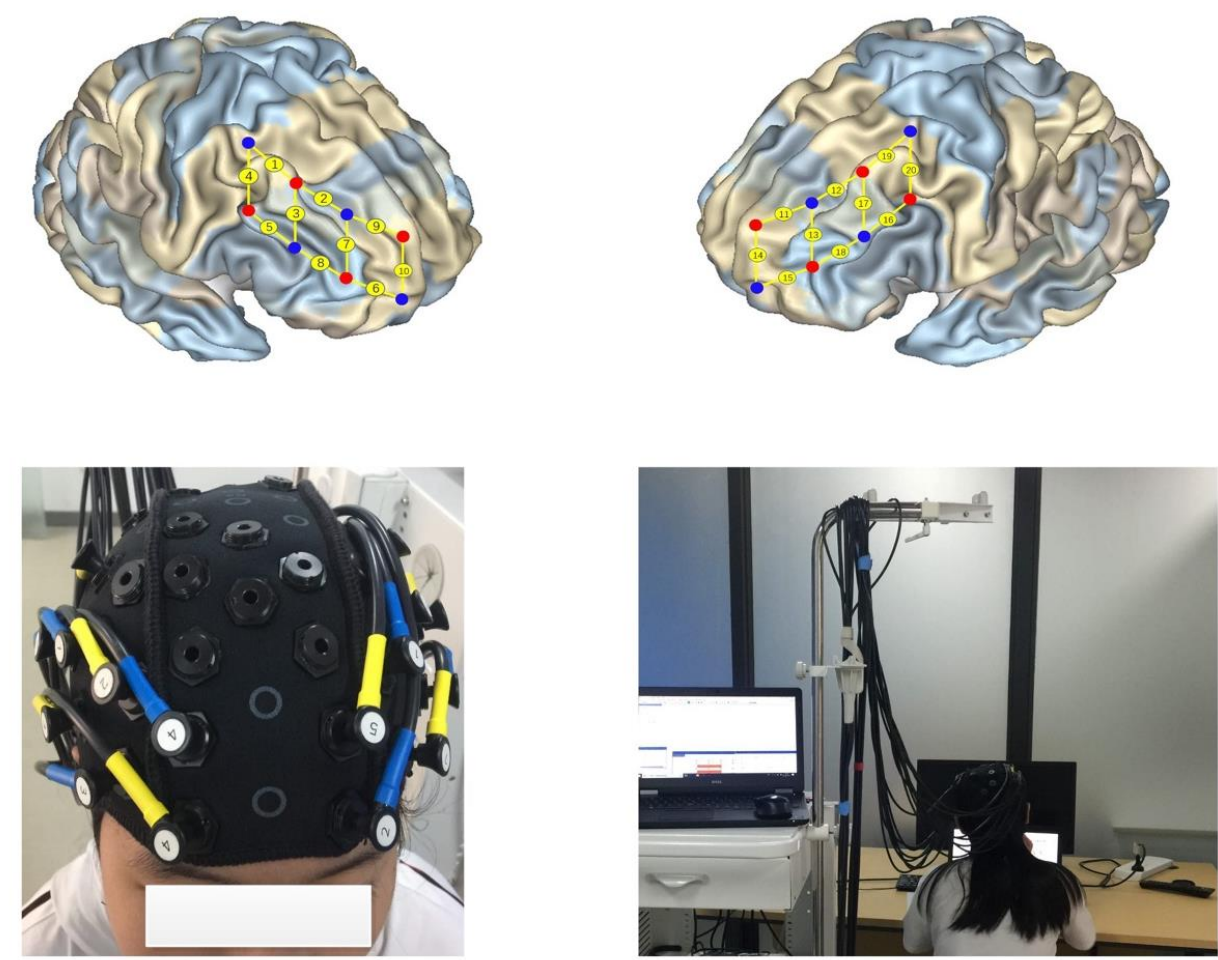

Notes. Sixteen probes (eight emitters: red and eight detectors: blue) were attached to the prefrontal areas forming 20 measurement channels (in yellow, only16 of which were finally analyzed in this study). The channels were placed following the international 10-20 system for EEG, with a $3 \mathrm{~cm}$ distance between each paired emitters and detectors. Channels 6, 9, 10, 11, 14 and 15 were located in the APFC (BA 10), channels $2,3,5,7,8,12,13,16,17$ and 18 were located in the DLPC (BA 46), and the channels 1, 4, 19 and 20 were located in the BA9 (excluded from this analysis due to poor signal quality).

\section{Procedure}

After arriving at the university laboratory, the adolescents and their parents were first briefed about the experiment, and the adolescents signed their written consent forms to the researcher. In the next step, they were asked to finish the questionnaire, including the media multitasking scale and executive function measures. Then, they were instructed about the task rules and given time to practice achieving the required accuracy (at least 70\%) on each task. Finally, they moved to the experiment room, put on the fNIRS equipment, and began the experiment. The data was collected during July to December 2018. After all data collection was finished, their completed questionnaires were analyzed first, and only the fNIRS data from those scoring in the upper and lower quartiles participants were further analyzed. Eventually, altogether 22 participants completed all of the measures in this study.

\section{Data Analysis}

\section{Self-Reported and Behavioral Data Analysis}

The questionnaire responses for each participant were summed to separated scores according to the original dimensions (working memory, inhibition, shifting, and dysexecutive function, respectively). The behavioral data were first calculated for each participant, and the groups were then compared using two-tailed t-tests. For the 2back task, we analyzed the false alarm rate, overall accuracy rate, and average reaction time. We also analyzed the overall accuracy rate and average reaction time for each participant for the Color Stroop task. We analyzed the switch cost for the number-letter task, such as average reaction time in switch trials minus average reaction time in continuous trials. For all reaction times, we removed all of the trials with response times shorter than $100 \mathrm{~ms}$.

\section{fNIRS Data Analysis}

The $\mathrm{HbO}$ levels between the two groups were compared during each task, but the $\mathrm{HbR}$ levels were not, as $\mathrm{HbO}$ is more sensitive than HbR to NIRS (Hoshi, 2007). The data from four channels (i.e., 1, 4, 19, and 20) were discarded 
due to the relatively low quality of the signals obtained from the participants. A bandpass filter $(0.02-0.2 \mathrm{~Hz})$ was first applied to the data to remove the longitudinal signal drift and physiological noise from the raw data using Homer 2. Then, for each participant, we further processed the data in Matlab 2013b to calculate the average $\mathrm{HbO}$ changes (i.e., $\triangle \mathrm{HbQ}$ ) in different channels and average three repeated blocks to produce the final value for each task. For all individual blocks, the baseline started at 10 s before each block onset. The task duration spanned is $4 \mathrm{~s}$ to $28 \mathrm{~s}$ (i.e., a total of 24s) after the onset of each block, which was regarded as the activation period during the task. Finally, two-tailed t-tests were conducted for each independent task using SPSS 26.0 to compare the average $\triangle \mathrm{HbO}$ in all channels between HMMs and LMMs groups.

\section{Reliability of the Analysis}

Although the current study was appropriately conducted, we noted that the sample size was relatively small. Therefore, we used three approaches to ensure the reliability of the analysis, as follows. First, for each significant result from the questionnaire, behavioral data, and fNIRS channels, we calculated the effect size (i.e., Cohen's d; Cohen, 1992). Following Cohen (1988), the criteria for this $d$ value were set as: follows: 0.8 (large), 0.5 (medium) and 0.2 (small). Second, the Bonferroni-based correction method (Shi et al., 2012) was used for multiple comparisons of multiple channels in fNIRS. The corrected $a^{*}=a / g^{*}, g^{*}=(g+1)-[1+(g-1) / C C](g=$ number of endpoints considered; ICC = sample-based intraclass correlation). The details of each correction are presented in the Results section. Third, we calculated the replication power by entering our current sample size and obtained the effect size into G*Power 3.1 (Faul et al., 2007) as one-tailed post hoc tests. A replication power above 0.80 is generally considered an acceptable level of power (Cohen, 1992).

\section{Results}

\section{Self-Reports and Behavioral Performance Results}

First of all, the correlations among the self-reports and behavioral performance were provided in Table 2. Then, the between-group differences in self-reports and behavioral performance were analyzed, and the results were presented in Table 3. First, the self-reported results indicated that the LMMs group scored significantly lower on the working memory $[t(20)=-3.213, p=.004, d=1.41]$, inhibition $[t(20)=-3.48, p=.002, d=1.50]$ and dysexecutive $[t(20)=-2.615, p=.017, d=1.11]$ measures than the HMMs group. However, no significant difference was found in the measure of shifting, $t(20)=-1.736, p=.098>.05$. All Cohen's $d$ values were above 0.8 , which indicated a large effect size (Cohen, 1988). The power analysis showed that all replication powers were above 0.80 , with the smallest being 0.80 (dysexecutive) and the largest being 0.96 (inhibition).

Table 2. Correlations Among MMS, Self-Reports, and Behavioral Performance for Both the LMMs and HMMs Groups.

\begin{tabular}{|c|c|c|c|c|c|c|c|c|c|c|c|}
\hline Measures & Details & 1 & 2 & 3 & 4 & 5 & 6 & 7 & 8 & 9 & 10 \\
\hline MMS & & - & & & & & & & & & \\
\hline \multirow[t]{3}{*}{ BRIEF 2} & $\begin{array}{l}\text { Working } \\
\text { memory }\end{array}$ & $.35(-.04)$ & - & & & & & & & & \\
\hline & Inhibition & $.62(.13)$ & $.67^{\star}\left(.81^{\star *}\right)$ & - & & & & & & & \\
\hline & Shifting & $.35(-.13)$ & $.75^{*}\left(.71^{* *}\right)$ & $.49(.53)$ & - & & & & & & \\
\hline DEX & & $.65^{*}(.10)$ & $.59\left(.75^{* \star}\right)$ & $.69^{*}\left(.72^{* *}\right)$ & $.57(.42)$ & - & & & & & \\
\hline \multirow[t]{2}{*}{ 2-back } & ACC & $-.24(.55)$ & $-.51(-.24)$ & $-.71^{*}(-.13)$ & $-.28(-.29)$ & $-.47(-.03)$ & - & & & & \\
\hline & RT & $.04(-.09)$ & $-.05(.14)$ & $.15(.06)$ & $-.47(.26)$ & $-.32(.20)$ & $-.33(.39)$ & - & & & \\
\hline \multirow[t]{2}{*}{ Color Stroop } & ACC & $-.43(-.37)$ & $-.12(.01)$ & $.08(-.06)$ & $.03(-.38)$ & $-.17(.18)$ & $-.39(.36)$ & $.23(-.34)$ & - & & \\
\hline & RT & $-.48(.09)$ & $-.43(-.11)$ & $-.29(-.26)$ & $-.86^{* *}(.31)$ & $-.52(-.22)$ & $.07(.19)$ & $.52\left(.73^{\star \star}\right)$ & $.16(-.50)$ & - & \\
\hline $\begin{array}{l}\text { Number- } \\
\text { letter Task }\end{array}$ & Switch-cost & $-.04(-.25)$ & $-.02(.23)$ & $-.19(-.20)$ & $.04(.39)$ & $.02(.11)$ & $-.13(-.19)$ & $.04(.20)$ & $.31(.15)$ & .13(.34) & - \\
\hline
\end{tabular}

Note. MMS = Media multitasking scale; BRIEF 2 = Behavior Rating Inventory of Executive Function (Second Edition); DEX = Dysexecutive Questionnaire; $A C C$ = Accuracy rate; RT = Reaction time; Switch-cost = Reaction time on switch trials minus reaction time on continuous trials; Correlations in parentheses are for the HMMs group.

${ }^{* *} p<.01,{ }^{*} p<.05$. 
Second, the behavioral results indicated no significant between-group differences in all three tasks, $p s .>0.05$. In particular, as shown in Table 2, the switch-costs, i.e., the reaction time on switch trials minus that on continuous trials, were negative for both the HMMs and LMMs groups. The one-sample t-test results indicated that the switchcost in the LMMs group was significantly lower than $0[t(9)=-2.31, p=.046]$, whereas that for the HMMs group was not significantly different from $0[t(11)=-1.83, p=.094]$.

Table 3. Differences Between the LMMs and HMMs Groups in the Questionnaire and Behavioral Performance.

\begin{tabular}{|c|c|c|c|c|c|c|c|c|c|c|}
\hline \multirow{2}{*}{ Measures } & \multirow{2}{*}{ Details } & \multicolumn{2}{|c|}{ LMMs $(n=10)$} & \multicolumn{2}{|c|}{ HMMs $(n=12)$} & \multirow{2}{*}{$F$} & \multirow{2}{*}{$t$} & \multirow{2}{*}{$d f$} & \multirow{2}{*}{$p$} & \multirow{2}{*}{$d$} \\
\hline & & $M$ & $S D$ & $M$ & $S D$ & & & & & \\
\hline \multirow[t]{3}{*}{ BRIEF 2} & Working memory & 13.50 & 2.64 & 18.42 & 4.19 & 0.98 & -3.21 & 20 & .004 & 1.41 \\
\hline & Inhibition & 11.50 & 2.64 & 15.75 & 3.02 & 0.08 & -3.48 & 20 & .002 & 1.50 \\
\hline & Shifting & 13.00 & 3.27 & 15.67 & 3.94 & 0.00 & -1.74 & 20 & .098 & 0.74 \\
\hline DEX & & 51.00 & 11.86 & 62.92 & 9.53 & 0.19 & -2.62 & 20 & .017 & 1.11 \\
\hline \multirow[t]{2}{*}{ 2-back } & ACC & 0.84 & 0.06 & 0.88 & 0.07 & 0.22 & -1.31 & 20 & .207 & 0.61 \\
\hline & RT & 873.95 & 242.97 & 842.19 & 177.35 & 1.23 & .35 & 20 & .727 & 0.15 \\
\hline \multirow[t]{2}{*}{ Color Stroop } & ACC & 0.96 & 0.02 & 0.96 & 0.04 & 1.92 & .05 & 20 & .960 & 0.00 \\
\hline & RT & 881.56 & 143.23 & 872.41 & 168.45 & 0.29 & .14 & 20 & .894 & 0.06 \\
\hline Number-letter Task & Switch-cost & -46.83 & 64.04 & -39.21 & 74.13 & 0.06 & -.26 & 20 & .801 & 0.11 \\
\hline
\end{tabular}

Note. BRIEF 2 = Behavior Rating Inventory of Executive Function (Second Edition); DEX = Dysexecutive Questionnaire; LMMs = Light media multitaskers; HMMs = Heavy media multitaskers; $\mathrm{ACC}=$ Accuracy rate; RT = Reaction time; Switch-cost = Reaction time on switch trials minus reaction time on continuous trials.

${ }^{* * *} p<.001,{ }^{* *} p<.01,{ }^{*} p<.05$.

\section{fNIRS Results}

First, we corrected the significance level a for multiple comparisons among 16 channels for each task. According to the Bonferroni-based correction method (Shi et al., 2012), the ICC (i.e., interclass correlation) for 2-back was .903 for these channels. Therefore, the corrected a for this 2-back task should be below .020. Similarly, the ICC for the Stroop task was .956 among these channels, and thus, the corrected a for this task should be below .030. For the number-letter task, the ICC was .881, and the corrected a for this task should be below .018 . Then, we analyzed each independent task separately. Only items with the abovementioned significance are reported in the following part.

The t-tests were conducted on all of the remaining 16 channels and the results indicated that the HbO changes of the HMMs were significantly higher than those of the LMMs in the 2-back and Color Stroop tasks. For the 2-back task, the significant between-group differences were found in the right hemisphere: $\operatorname{Ch} 2[t(20)=-4.184, p<.001$, $d=1.78]$, Ch3 [t(20) $=-2.536, p=.020, d=1.10], \operatorname{Ch} 7[t(20)=-3.798, p=.001, d=1.57]$, Ch8 [t(20) $=-2.650, p=.015$, $d=1.17]$ and Ch9 [t(20) $=-3.566, p=.002, d=1.53]$, and left hemisphere: Ch13 [t(20) $=-3.273, p=.004, d=1.40]$. For the Stroop task, the significant between-group differences were found in the right hemisphere: Ch8 $[t(20)=-2.744, p=.013, d=1.15]$, and left hemisphere: Ch12 [t(20) $=-2.755, p=.012, d=1.17]$. All Cohen's d values were also above 0.8 , which indicated a large effect size (Cohen, 1988). The power analysis showed that all replication powers were above 0.80 , with the smallest being 0.80 (2-back, Ch3) and largest being 0.99 (2-back; Ch2). However, for the number-letter task, there were no significant between-group differences for any of the channels, and the smallest (but non-significant) $p$ value was found in the right hemisphere: $\operatorname{Ch} 3[t(20)=-1.858, p=.078]$. All of the above-reported significant channels are shown in Figure 3. 
Figure 3. fNIRS Results for Significant Channels: (a) Significant Channels for the 2-Back Task, (b) Significant Channels for Color Stroop Task.
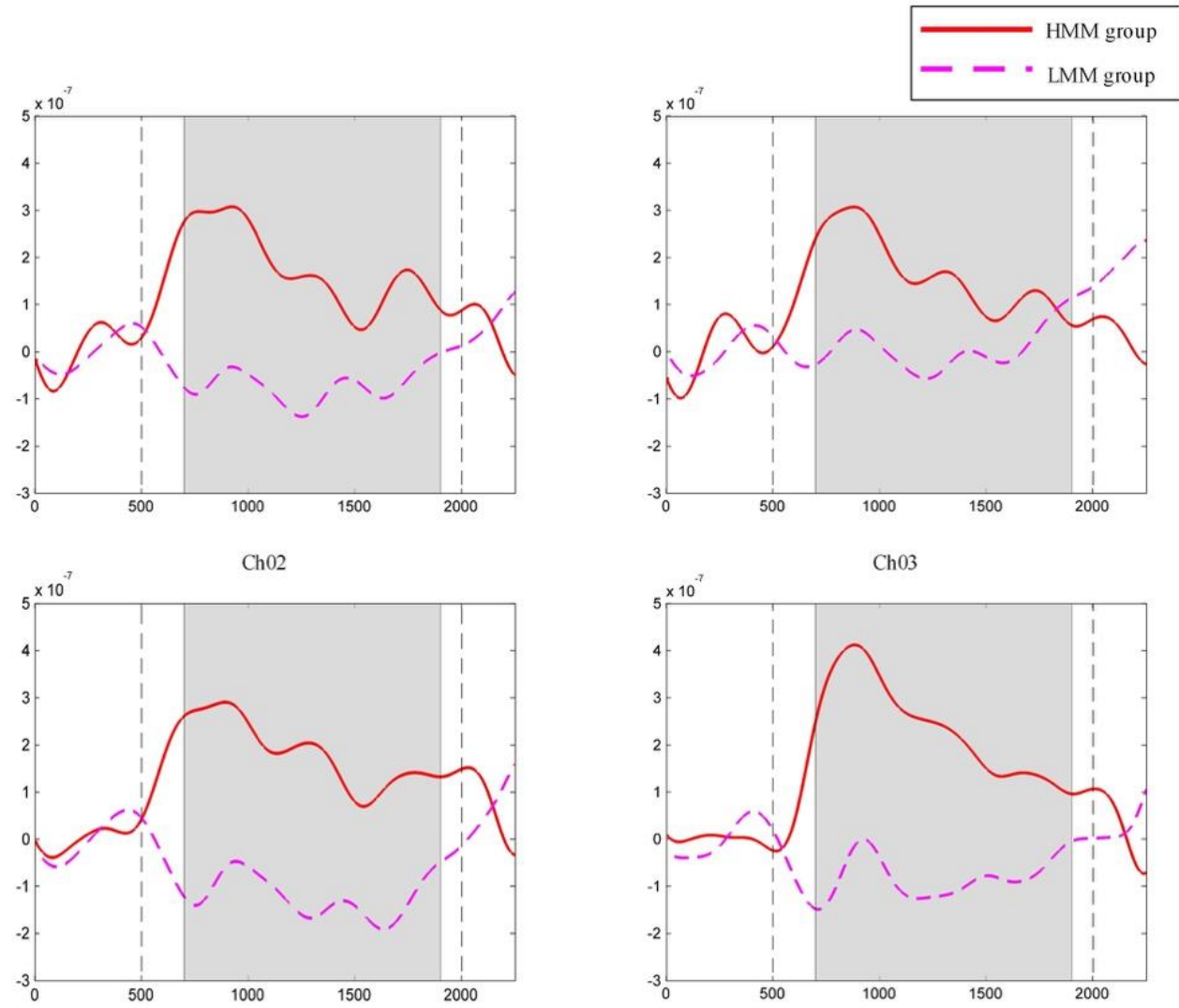

Ch07
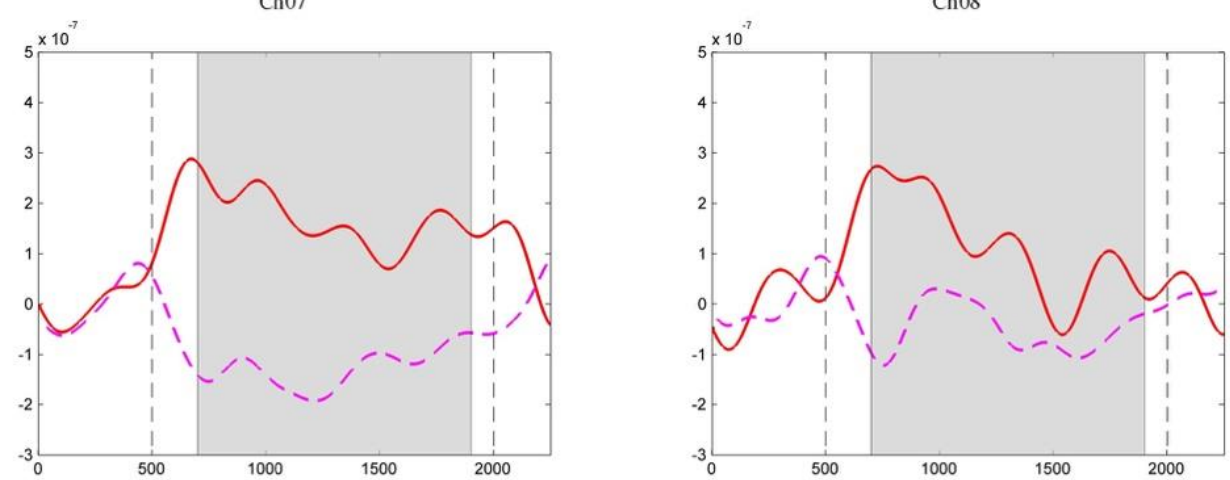

Ch09

Ch 13

(a) Significant channels for 2-back task
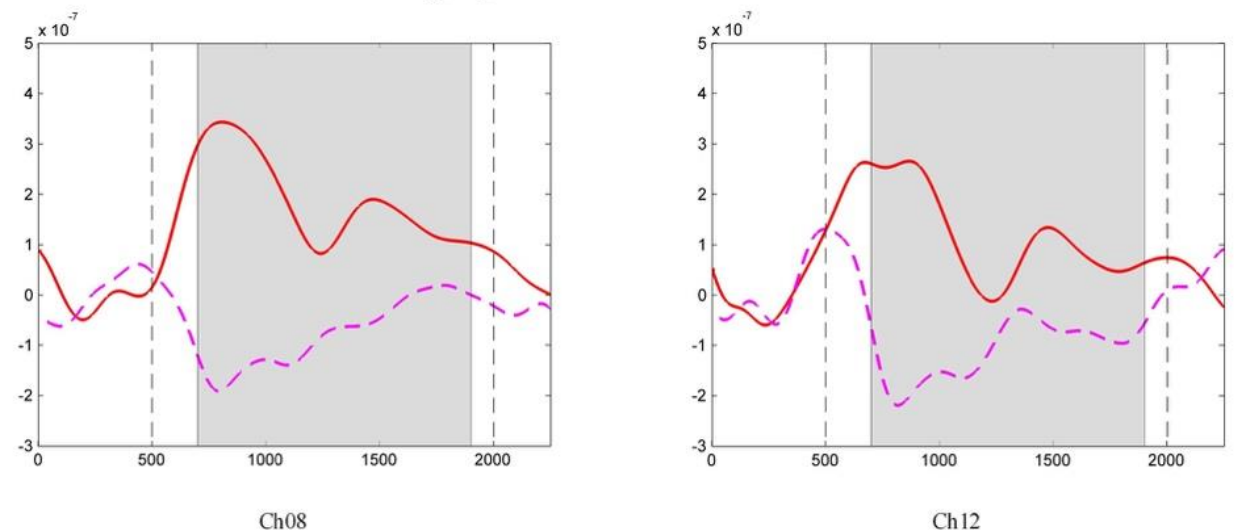

(b) Significant channels for color stroop task 


\section{Discussion}

This study established a triangulation of data sources - the self-reported questionnaire, behavioral tasks, and fNIRS - to examine the differences in executive function between heavy/high media multitaskers (HMMs) and light/low media multitaskers (LMMs). This transdisciplinary approach allowed us to further explore the neural differences between HMMs and LMMs and compare with corresponding observational and behavioral differences. In this section, we discussed the triangulated findings and their implications.

\section{HMMs Versus LMMs: The 3-Level Differences and the Neurocognitive Causes}

First, the self-reported results showed that the HMMs group performed worse than the LMMs group on executive function measures, including working memory, inhibition and general executive function. Therefore, Hypothesis 1 was generally supported. This finding is consistent with that of Baumgartner et al. (2014), who used questionnaire measures of media multitaskers' behaviour in daily life and found that media multitasking was highly correlated with problems in working memory and inhibition performance. In addition, the present differences between HMMs and LMMs in general executive function are also consistent with previous studies indicating that HMMs scored higher on impulsivity and sensation seeking (Luo \& Liang, 2018; Magen, 2017; Sanbonmatsu et al., 2013).

Second, the behavioral study revealed no significant between-group differences in working memory and inhibition. In this case, Hypothesis 2 was not supported in the current study. This finding is also inconsistent with the previous studies in which HMMs generally performed worse than LMMs on cognitive tasks such as n-back (Cardoso-Leite et al., 2016; Ophir et al., 2009; Ralph \& Smilek, 2017; Wiradhany \& Nieuwenstein, 2017). who used questionnaire measures of media multitaskers' behaviour in daily life and found that media multitasking was highly correlated with problems in working memory and inhibition performance. This finding may be attributable to one of two factors. First, the finding of no differences between HMMs and LMMs from this study might be accurate and consistent with previous studies (for reviews, see Uncapher \& Wagner, 2018; Wiradhany \& Nieuwenstein, 2017). Second, it might be related to the different task conditions between this and previous studies. Specifically, before starting the experiment, the participants in this study were allowed to practice as many tails as they needed to achieve a $70 \%$ accuracy. Although for some participants, their accuracy might have decreased during the formal experiment, such effects were not expected to be large. Moreover, unlike in the previous studies focusing on behavioral performance (Ophir et al., 2009; Wiradhany \& Nieuwenstein, 2017), the number of trials in each task was limited to enable fNIRS data collection in this study. Therefore, these task modifications may have affected their behavioral performance, which would complicate the interpretation of the non-significant findings. However, the two factors are interrelated, and the different experimental conditions may also have contributed to the conflicting results of previous studies.

Third, the fNIRS study showed greater activity in some prefrontal areas of the HMMs during the 2-back and Color Stroop tasks. Thus, Hypothesis 3 was also partially supported. For the 2-back task, six channels located in both right (i.e., Ch2, 3, 7, 8, 9; BA46 and BA10) and left hemispheres (i.e., Ch13; BA46) were more activated in HMMs than in LMMs. For the Color Stroop task, two channels, one located in the right hemisphere (i.e., Ch8; BA46) and one in the left hemisphere (i.e., Ch12; BA46), were also found to be more activated in the HMMs. This is the first study to report the neural differences between HMMs and LMMs while performing working memory (i.e., 2-back) and inhibition (i.e., Color Stroop) tasks. This finding provides neuroimaging evidence to support the conclusion of previous studies that the neural differences between HMMs and LMMs may be located in prefrontal areas (Loh \& Kanai, 2014; Moisala et al., 2016). In addition, this study revealed that in both the 2-back and Color Stroop tasks, BA46 played an important role in distinguishing LMMs and HMMs. This finding is consistent with previous studies (e.g., J. Ducan \& Owen, 2000), which found that the DLPFC, including BA46, was highly responsible for executive function across various task paradigms. Besides, BA46 has also been shown to be involved in sustaining attention and self-control (Corbetta \& Shulman, 2002; Smith et al., 2011). Furthermore, it has been widely suggested that media multitasking may have a negative effect on adolescents' attention, executive function and self-control (e.g., Ophir et al., 2009; Xu et al., 2016); accordingly, its adverse effects might arise in the relevant brain areas including BA46. This implies that we should pay more attention to the effects of media multitasking, which is becoming ever increasingly prevalent, on the development of this brain area (i.e., BA46). Besides, we should also be cautious with 
the possible vicious loop effect - increasing the susceptibility to media multitasking, which will impact the EFrelated brain areas.

Last but not least, the self-reported questionnaire, behavioral task, and fNIRS results in this study all indicated no significant differences between the HMMs and LMMs in shifting ability. On the one hand, this is inconsistent with the previous findings of the negative effect of media multitasking on shifting ability (e.g., Ophir et al., 2009). There are two possible explanations for this result. First, the non-significant findings on the behavioral task and fNIRS might due to the design of the present study. For example, all participants finished the cognitive tasks with fNIRS in the same order, and the shifting task (i.e., the Number-letter task) was the last one. Therefore, the task order might have affected the findings on shifting ability. Second, the non-significant results, which were consistently found in all three measurements, i.e., self-reported questionnaire, behavioral task, and fNIRS, indicated that in our sample, the HMMs and LMMs were not different in terms of the shifting aspect. On the other hand, there is an emerging pattern of evidence showing no differences between HMMs and LMMs on shifting, or even no association between media multitasking score and shifting (Cardoso-Leite et al., 2016; Gorman \& Green, 2016; Minear et al., 2013; Seddon et al., 2018). For example, Rogobete et al. (2020) considered media multitasking with media activities and non-media activities, and found that the relationship between media multitasking and shifting was not significant; the researchers further doubted that there was a non-linear relationship between media multitasking and executive function. This finding provides strong evidence to query whether there are significant between-group differences between HMMs and LMMs on shifting aspect with methodological triangulation; and replication studies are therefore needed in the future. Nevertheless, the behavioral results indicated that LMMs might take less time to finish switch trials than repeated trials, but HMMs did not differ on repeated and switch trials. In other words, only the LMMs group performed better on switch trials than repeated trials. This new evidence indicates that LMMs may have better shifting ability. However, it remains unclear why LMMs respond to switch trials faster than repeated trials. Because the use of this Number-letter task has not been widely reported either among adolescents or in the comparisons between HMMs and LMMs in the Chinese context, more efforts should be dedicated to this aspect in future research. In particular, further transdisciplinary studies on the shifting ability using other task paradigms are also needed in comparisons of HMMs and LMMs.

However, caution should be taken regarding the directionality of the findings. It is possible that executive function develops differently across adolescents, and lower executive function (or greater distractibility) might increase the likelihood of engaging in media multitasking. Future studies could use a longitudinal design to clarify whether there is a causal relationship between media multitasking and executive function.

\section{HMMs Versus LMMs: Is Higher Activation Always Better?}

This study found that HMMs showed impaired performance on working memory and inhibition than LMMs, while their DLPFC and APFC areas were significantly more activated. In the existing literature, extensive brain activation in these two areas has been most frequently identified with a better ability to perform the corresponding executive function and often associated with a better behavioral result (e.g., Cutini et al., 2008; Ehlis et al., 2008; Perlman et al., 2016). Although there were no differences in their behavioral performances in this study, the HMMs still showed lower executive function than the LMMs. Nevertheless, the higher activation that we found in the HMMs group was consistent with the study by Moisala et al.'s (2016), which also reported a higher brain activation among HMMs in the right prefrontal area. The question, then, is why the HMMs had a higher brain activation than the LMMs.

First, as the previous review indicated, HMMs might need more effort to focus and achieve the same result in cognitive tasks (see Uncapher \& Wagner, 2018). That argument is supported by this study's neuroimaging evidence from Chinese adolescents. Second, as the media have penetrated daily lives, it is possible that our brains, especially those of the young, have been shaped by media activities, including media multitasking (Gazzaley \& Rosen, 2016; Rothbart \& Posner, 2015). HMMs engage in more media activities, and their brains are often more restless, and possibly over-used. This effect may persist even during cognitive tasks. In addition, it might also be the case that the brains of HMMs are less effective than those of LMMs during task performance. For example, Morgan and D'Mello (2016) argued that multitasking could lead to exhaustion in working memory and attention resources, and Levitin (2015) showed that such exhaustion could harm the brain. Besides, some researchers have also expressed concern that multitasking - switching between multiple sources of information - might trigger a 
symptom known as "iDisorder" (Carrier et al., 2015; Rosen, 2012; Rosen et al., 2013). In their opinion, the modern brain is prone to various psychological symptoms, such as stress, sleeplessness and compulsive device checking, due to media and technology overuse in daily life, including but not limited to media multitasking. Finally, the finding that the brains of HMMs worked less effectively than expected implies that we should pay more attention to the long-term effects of media multitasking on adolescents' neurological development in the era in which the media, especially social media, have penetrated their lives both inside and outside school (Lu et al., 2019; Luo, Liang, et al., 2020).

\section{Conclusion}

This multiple-approach study found no significant differences between the HMMs and LMMs groups' performance on three behavioral tasks, indicating that both groups performed at the same behavioral level. However, the selfreported results indicated that the adolescent HMMs displayed a more impaired performance in executive function than the adolescent LMMs. In addition, the HMMs group also showed greater activation in the brain areas responsible for executive function during the 2-back and Color Stroop tasks than the LMMs group. These results jointly indicated that the HMMs group required more brain activation to achieve the same behavioral performance as the LMMs. This finding implies that media multitasking might be associated with reduced effectiveness in the brain areas responsible for executive function.

Although this study provides more comprehensive evidence on the association between media multitasking and executive function than was previously available, it has some limitations. First, the number of participants was very limited, and they might not represent all Chinese adolescents. Future studies should use a more suitable sample by recruiting more representative participants and increasing the sample size. Second, the effect of task order was not controlled in this study. Specifically, all participants finished the 2-back, Color Stroop, and Numberletter tasks in the same order. Future research should explore whether the task order can affect their brain activation. Third, only one task was used to examine each executive function domain, i.e., 2-back for working memory, Color Stroop for inhibition, and Number-letter for shifting. Future studies should also consider using different tasks, especially tasks for shifting, to further compare the brain activation between HMMs and LMMs. In addition, this study found no significant differences between HMMs and LMMs in the self-reported measure of shifting; future studies should pay attention to this aspect and provide more evidence regarding the shifting ability. Fourth, this study allowed the participants to practice until they achieved a $70 \%$ accuracy threshold to ensure that they fully understood the rules; thus, they could activate the relevant brain areas corresponding to executive function. Future studies should consider avoiding the practice effects, such as by giving participants enough rests before formal testing. Fifth, this study only examined the differences between HMMs and LMMs and did not consider the full continuum of media multitaskers. Future studies should consider including the moderate or average media multitaskers (Shin et al., 2020), which represented the group between HMMs and LMMs, into this kind of transdisciplinary comparison. Last, we calculated the effect size and replication power at the end of the data analysis because we did not have sufficient data to decide beforehand. However, future studies could start from expected effect sizes and determine the sample size needed. Nevertheless, this study has successfully identified between-group differences in the activation of brain areas responsible for executive function, providing neuroimaging evidence of the negative association of media multitasking on executive function in adolescents.

\section{Acknowledgements}

This article is extracted from the first author's PhD thesis. This research was partially supported by the Shenzhen Fundamental Research Project (JCYJ20170412111316339) and the Shenzhen Talent Peacock Team Plan (827000083). The authors would like to thank the late Associate Professor Dr. Jingyan Lu at the Faculty of Education, the University of Hong Kong, for her great support to this PhD project in the early stage. We would also like to thank Dr. Fan Zhu (Frank) for his advice to the fNIRS experiment, and Mr. Jinfeng Yang at Shenzhen University for his assistant in preliminary data analysis. Besides, we are also very appreciated the reviewers for taking time to review this manuscript and providing valuable suggestions for improvement. Last but not least, the authors would extend our sincere thanks to all the teachers, parents, and students for their help during this study. 


\section{Author Contribution}

$J \mathrm{~L}$ conducted the research, performed the data analysis and write the manuscript. $\mathrm{HL}$ supervised the research, refined the research design and revised the manuscript. PY supervised the research. CC supervised and supported this research.

\section{References}

Basura, G. J., Hu, X.-S., Juan, J. S., Tessier, A.-M., \& Kovelman, I. (2018). Human central auditory plasticity: A review of functional near-infrared spectroscopy (fNIRS) to measure cochlear implant performance and tinnitus perception. Laryngoscope Investigative Otolaryngology, 3(6), 463-472. https://doi.org/10.1002/lio2.185

Baumgartner, S. E., van der Schuur, W. A., Lemmens, J. S., \& te Poel, F. (2018). The relationship between media multitasking and attention problems in adolescents: Results of two longitudinal studies. Human Communication Research, 44(1), 3-30. https://doi.org/10.1093/hcre.12111

Baumgartner, S. E., Weeda, W. D., van der Heijden, L. L., \& Huizinga, M. (2014). The relationship between media multitasking and executive function in early adolescents. The Journal of Early Adolescence, 34(8), 1120-1144. https://doi.org/10.1177/0272431614523133

Becker, M. W., Alzahabi, R., \& Hopwood, C. J. (2013). Media multitasking is associated with symptoms of depression and social anxiety. Cyberpsychology, Behavior, and Social Networking, 16(2), 132-135.

https://doi.org/10.1089/cyber.2012.0291

Burgess, P. W., Alderman, N., Evans, J., Emslie, H., \& Wilson, B. A. (1998). The ecological validity of tests of executive function. Journal of the International Neuropsychological Society, 4(6), 547-558.

https://doi.org/10.1017/S1355617798466037

Cain, M. S., Leonard, J. A., Gabrieli, J. D. E., \& Finn, A. S. (2016). Media multitasking in adolescence. Psychonomic Bulletin \& Review, 23(6), 1932-1941. https://doi.org/10.3758/s13423-016-1036-3

Cardoso-Leite, P., Kludt, R., Vignola, G., Ma, W. J., Green, C. S., \& Bavelier, D. (2016). Technology consumption and cognitive control: Contrasting action video game experience with media multitasking. Attention, Perception, and Psychophysics, 78(1), 218-241. https://doi.org/10.3758/s13414-015-0988-0

Carrier, L. M., Rosen, L. D., Cheever, N. A., \& Lim, A. F. (2015). Causes, effects, and practicalities of everyday multitasking. Developmental Review, 35, 64-78. https://doi.org/10.1016/j.dr.2014.12.005

Chan, R. C. K. (2001). Dysexecutive symptoms among a non-clinical sample: A study with the use of the Dysexecutive Questionnaire. British Journal of Psychology, 92(3), 551-565.

https://doi.org/10.1348/000712601162338

Chen, X., Wang, Y., Tao, D., Jiang, L., \& Li, S. (2021). Antecedents of smartphone multitasking: Roles of demographics, personalities and motivations. Internet Research. Advance online publication.

https://doi.org/10.1108/intr-09-2019-0388

Cohen, J. (1988). Statistical power analysis for the behavioral sciences (2nd ed.). Lawrence Erlbaum Associates.

Cohen, J. (1992). A power primer. Psychological Bulletin, 112(1), 155-159. https://doi.org/10.1037/0033-

2909.112.1.155

Corbetta, M., \& Shulman, G. L. (2002). Control of goal-directed and stimulus-driven attention in the brain. Nature Reviews Neuroscience, 3(3), 201-215. https://doi.org/10.1038/nrn755 
Cutini, S., Scatturin, P., Menon, E., Bisiacchi, P. S., Gamberini, L., Zorzi, M., \& Dell'Acqua, R. (2008). Selective activation of the superior frontal gyrus in task-switching: An event-related fNIRS study. Neurolmage, 42(2), 945955. https://doi.org/10.1016/j.neuroimage.2008.05.013

Diamond, A. (2013). Executive functions. Annual Review of Psychology, 64, 135-168.

https://doi.org/10.1146/annurev-psych-113011-143750

Dreher, J.-C., Koechlin, E., Tierney, M., \& Grafman, J. (2008). Damage to the fronto-polar cortex is associated with impaired multitasking. PLOS ONE, 3(9), Article e3227. https://doi.org/10.1371/journal.pone.0003227

Ducan, A., Meek, J. H., Clemence, M., Elwell, C. E., Fallon, P., Tyszczuk, L., Cope, M., \& Delpy, D. T. (1996). Measurement of cranial optical path length as a function of age using phase resolved near infrared spectroscopy. Pediatric Research, 39(5), 889-894. http://www.ncbi.nlm.nih.gov/pubmed/9255710

Ducan, J., \& Owen, A. M. (2000). Common regions of the human frontal lobe recruited by diverse cognitive demands. Trends in Neuroscience, 23(10), 475-483. https://doi.org/10.1016/S0166-2236(00)01633-7

Dumontheil, I. (2016). Adolescent brain development. Current Issues in Behavioral Sciences, 10, 39-44. https://doi.org/10.1016/j.cobeha.2016.04.012

Ehlis, A.-C., Bähne, C. G., Jacob, C. P., Herrmann, M. J., \& Fallgatter, A. J. (2008). Reduced lateral prefrontal activation in adult patients with attention-deficit/hyperactivity disorder (ADHD) during a working memory task: A functional near-infrared spectroscopy (fNIRS) study. Journal of Psychiatric Research, 42(13), 1060-1067. https://doi.org/10.1016/j.jpsychires.2007.11.011

Faul, F., Erdfelder, E., Lang, A.-G., \& Buchner, A. (2007). G*Power 3: A flexible statistical power analysis program for the social, behavioral, and biomedical sciences. Behavior Research Methods, 39(2), 175-191.

https://doi.org/10.3758/BF03193146

Foehr, U. G. (2006). Media multitasking among American youth: Prevalence, predictors, and pairings. The Henry J. Kaiser Family Foundation. http://kff.org/other/media-multitasking-among-american-youth-prevalencepredictors/

Gazzaley, A., \& Rosen, L. D. (2016). The distracted mind: Ancient brains in a high-tech world. MIT Press.

Gioia, G. A., Isquith, P. K., Guy, S. C., \& Kenworthy, L. (2015). Behavior Rating Inventory of Executive Function, Second Edition (BRIEF ${ }^{\circledR}$ 2). PAR, Inc. https://www.parinc.com/products/pkey/24

Gorman, T. E., \& Green, C. S. (2016). Short-term mindfulness intervention reduces the negative attentional effects associated with heavy media multitasking. Scientific Reports, 6, Article 24542.

https://doi.org/10.1038/srep24542

Herold, F., Wiegel, P., Scholkmann, F., \& Müller, N. G. (2018). Applications of functional near-infrared spectroscopy (fNIRS) neuroimaging in exercise-cognition science: A systematic, methodology-focused review. Journal of Clinical Medicine, 7(12), Article 466. https://doi.org/10.3390/jcm7120466

Hoshi, Y. (2007). Functional near-infrared spectroscopy: Current status and future prospects. Journal of Biomedical Optics, 12(6), Article 062106. https://doi.org/10.1117/1.2804911

Huizinga, M., Dolan, C. V., \& van der Molen, M. W. (2006). Age-related change in executive function: Developmental trends and a latent variable analysis. Neuropsychologia, 44(11), 2017-2036.

https://doi.org/10.1016/j.neuropsychologia.2006.01.010 
Huizinga, M., \& Smidts, D. P. (2010). Age-related changes in executive function: A normative study with the Dutch version of the Behavior Rating Inventory of Executive Function (BRIEF). Child Neuropsychology, 17(1), 51-66. https://doi.org/10.1080/09297049.2010.509715

Koike, S., Takizawa, R., Nishimura, Y., Kinou, M., Kawasaki, S., \& Kasai, K. (2013). Reduced but broader prefrontal activity in patients with schizophrenia during n-back working memory tasks: A multi-channel near-infrared spectroscopy study. Journal of Psychiatric Research, 47(9), 1240-1246.

https://doi.org/10.1016/j.jpsychires.2013.05.009

Laguë-Beauvais, M., Brunet, J., Gagnon, L., Lesage, F., \& Bherer, L. (2013). A fNIRS investigation of switching and inhibition during the modified Stroop task in younger and older adults. Neurolmage, 64, 485-495.

https://doi.org/10.1016/j.neuroimage.2012.09.042

León-Domínguez, U., Martín-Rodríguez, J. F., \& León-Carrión, J. (2015). Executive n-back tasks for the neuropsychological assessment of working memory. Behavioural Brain Research, 292, 167-173. https://doi.org/10.1016/j.bbr.2015.06.002

Levitin, D. J. (2015, January 18). Why the modern world is bad for your brain. The Guardian.

https://www.theguardian.com/science/2015/jan/18/modern-world-bad-for-brain-daniel-j-levitin-organized-mindinformation-overload

Loh, K. K., \& Kanai, R. (2014). Higher media multi-tasking activity is associated with smaller gray-matter density in the anterior cingulate cortex. PLOS ONE, 9(9), Article e106698. https://doi.org/10.1371/journal.pone.0106698

Lu, J., Luo, J., Liang, L., \& Jing, M. (2019). Measuring adolescents' social media behavior outside and inside of school: Development and validation of two scales. Journal of Educational Computing Research, 57(5), 1108-1130. https://doi.org/10.1177/0735633118786851

Luo, J., \& Liang, L. (2018). Exploring the relationships among university students' media multitasking, personality and academic performance: A quantitative study [Paper presentation]. International Scientific Conference: Lodging the Theory in Social and Educational Practice. https://goo.gl/RbjvjH

Luo, J., Liang, L., \& Li, H. (2020). The divergent roles of social media in adolescents' academic performance. Journal of Research in Childhood Education, 34(2), 167-182. https://doi.org/10.1080/02568543.2019.1703124

Luo, J., Sun, M., Yeung, P., \& Li, H. (2018). Development and validation of a scale to measure media multitasking among adolescents: Results from China. Children and Youth Services Review, 95, 377-383.

https://doi.org/10.1016/j.childyouth.2018.10.044

Luo, J., Yeung, P., \& Li, H. (2020a). The relationship among media multitasking, academic performance and selfesteem in Chinese adolescents: The cross-lagged panel and mediation analyses. Children and Youth Services Review, 117, Article 105308. https://doi.org/10.1016/j.childyouth.2020.105308

Luo, J., Yeung, P., \& Li, H. (2020b). Relationship between media multitasking and self-esteem among Chinese adolescents: Mediating roles of peer influence and family functioning. Journal of Child and Family Studies, 29(5), 1391-1401. https://doi.org/10.1007/s10826-019-01613-3

Magen, H. (2017). The relations between executive functions, media multitasking and polychronicity. Computers in Human Behavior, 67, 1-9. https://doi.org/10.1016/j.chb.2016.10.011

Minear, M., Brasher, F., McCurdy, M., Lewis, J., \& Younggren, A. (2013). Working memory, fluid intelligence, and impulsiveness in heavy media multitaskers. Psychonomic Bulletin \& Review, 20(6), 1274-1281.

https://doi.org/10.3758/s13423-013-0456-6 
Miyake, A., Friedman, N. P., Emerson, M. J., Witzki, A. H., Howerter, A., \& Wager, T. D. (2000). The unity and diversity of executive functions and their contributions to complex "frontal lobe" tasks: A latent variable analysis. Cognitive Psychology, 41(1), 49-100. https://doi.org/10.1006/cogp.1999.0734

Moisala, M., Salmela, V., Hietajärvi, L., Salo, E., Carlson, S., Salonen, O., Lonka, K., Hakkarainen, K., Salmela-Aro, K., \& Alho, K. (2016). Media multitasking is associated with distractibility and increased prefrontal activity in adolescents and young adults. Neurolmage, 134, 113-121. https://doi.org/10.1016/j.neuroimage.2016.04.011

Morgan, B., \& D'Mello, S. K. (2016). The influence of positive vs. negative affect on multitasking. Acta Psychologica, 170, 10-18. https://doi.org/10.1016/j.actpsy.2016.06.006

Moriguchi, Y., \& Hiraki, K. (2013). Prefrontal cortex and executive function in young children: A review of NIRS studies. Frontiers in Human Neuroscience, 7, Article 867. https://doi.org/10.3389/fnhum.2013.00867

Murphy, K., \& Creux, O. (2021). Examining the association between media multitasking, and performance on working memory and inhibition tasks. Computers in Human Behavior, 114, Article 106532.

https://doi.org/10.1016/j.chb.2020.106532

Ophir, E., Nass, C., \& Wagner, A. D. (2009). Cognitive control in media multitaskers. PNAS, 106(37), 15583-15587. https://doi.org/10.1073/pnas.0903620106

Parker, J. K. (2012). Prefrontal oxygenation during executive tasks in children with developmental coordination disorder [Master's thesis, lowa State University]. Iowa State University Digital Repository.

https://lib.dr.iastate.edu/etd/12428

Parry, D. A., \& le Roux, D. B. (2019). Media multitasking and cognitive control: A systematic review of interventions. Computers in Human Behavior, 92, 316-327. https://doi.org/10.1016/j.chb.2018.11.031

Perlman, S. B., Huppert, T. J., \& Luna, B. (2016). Functional near-infrared spectroscopy evidence for development of prefrontal engagement in working memory in early through middle childhood. Cerebral Cortex, 26(6), 27902799. https://doi.org/10.1093/cercor/bhv139

Ralph, B. C. W., \& Smilek, D. (2017). Individual differences in media multitasking and performance on the n-back. Attention, Perception, and Psychophysics, 79(2), 582-592. https://doi.org/10.3758/s13414-016-1260-y

Roca, M., Torralva, T., Gleichgerrcht, E., Woolgar, A., Thompson, R., Duncan, J., \& Manes, F. (2011). The role of Area 10 (BA10) in human multitasking and in social cognition: A lesion study. Neuropsychologia, 49(13), 35253531. https://doi.org/10.1016/j.neuropsychologia.2011.09.003

Rogobete, D. A., lonescu, T., \& Miclea, M. (2020). The relationship between media multitasking behavior and executive function in adolescence: A replication study. The Journal of Early Adolescence. Advance online publication. https://doi.org/10.1177/0272431620950478

Rosen, L. D. (2012). iDisorder: Understanding our obsession with technology and overcoming its hold on us. Palgrave Macmillan.

Rosen, L. D., Whaling, K., Rab, S., Carrier, L. M., \& Cheever, N. A. (2013). Is Facebook creating "iDisorders"? The link between clinical symptoms of psychiatric disorders and technology use, attitudes and anxiety. Computers in Human Behavior, 29(3), 1243-1254. https://doi.org/10.1016/j.chb.2012.11.012

Rothbart, M. K., \& Posner, M. I. (2015). The developing brain in a multitasking world. Developmental Review, 35, 42-63. https://doi.org/10.1016/j.dr.2014.12.006 
Sanbonmatsu, D. M., Strayer, D. L., Medeiros-Ward, N., \& Watson, J. M. (2013). Who multi-tasks and why? Multitasking ability, perceived multi-tasking ability, impulsivity, and sensation seeking. PLOS ONE, 8(1), Article e54402. https://doi.org/10.1371/journal.pone.0054402

Schroeter, M. L., Zysset, S., Wahl, M., \& von Cramon, D. Y. (2004). Prefrontal activation due to Stroop interference increases during development-an event-related fNIRS study. Neurolmage, 23(4), 1317-1325.

https://doi.org/10.1016/j.neuroimage.2004.08.001

Seddon, A. L., Law, A. S., Adams, A.-M., \& Simmons, F. R. (2018). Exploring the relationship between executive functions and self-reported media-multitasking in young adults. Journal of Cognitive Psychology, 30(7), 728-742. https://doi.org/10.1080/20445911.2018.1525387

Shi, Q., Pavey, E. S., \& Carter, R. E. (2012). Bonferroni-based correction factor for multiple, correlated endpoints. Pharmaceutical Statistics, 11(4), 300-309. https://doi.org/10.1002/pst.1514

Shih, S.-I. (2013). A null relationship between media multitasking and well-being. PLoS ONE, 8(5), Article e64508. https://doi.org/10.1371/journal.pone.0064508

Shin, M., Linke, A., \& Kemps, E. (2020). Moderate amounts of media multitasking are associated with optimal task performance and minimal mind wandering. Computers in Human Behavior, 111, Article 106422.

https://doi.org/10.1016/j.chb.2020.106422

Shin, M., Webb, A., \& Kemps, E. (2019). Media multitasking, impulsivity and dual task ability. Computers in Human Behavior, 92, 160-168. https://doi.org/10.1016/j.chb.2018.11.018

Smith, A. B., Halari, R., Giampetro, V., Brammer, M., \& Rubia, K. (2011). Developmental effects of reward on sustained attention networks. Neurolmage, 56(3), 1693-1704. https://doi.org/10.1016/j.neuroimage.2011.01.072

Steinberg, L. (2005). Cognitive and affective development in adolescence. Trends in Cognitive Sciences, 9(2), 69-74. https://doi.org/10.1016/j.tics.2004.12.005

Strangman, G., Boas, D. A., \& Sutton, J. P. (2002). Non-invasive neuroimaging using near-infrared light. Biological Psychiatry, 52(7), 679-693. https://doi.org/10.1016/S0006-3223(02)01550-0

Uncapher, M. R., Lin, L., Rosen, L. D., Kirkorian, H. L., Baron, N. S., Bailey, K., Cantor, J., Strayer, D. L., Parsons, T. D., \& Wagner, A. D. (2017). Media multitasking and cognitive, psychological, neural, and learning differences. Pediatrics, 140(Suppl. 2), S62-S66. https://doi.org/10.1542/peds.2016-1758D

Uncapher, M. R., \& Wagner, A. D. (2018). Minds and brains of media multitaskers: Current findings and future directions. PNAS, 115(40), 9889-9896. https://doi.org/10.1073/pnas.1611612115

Wallis, C. (2010). The impacts of media multitasking on children's learning and development: Report from a research seminar. The Joan Ganz Cooney Center and Stanford University.

Wilson, B. A., Evans, J. J., Emslie, H., Alderman, N., \& Burgess, P. (1998). The development of an ecologically valid test for assessing patients with a dysexecutive syndrome. Neuropsychological Rehabilitation, 8(3), 213-228.

https://doi.org/10.1080/713755570

Wiradhany, W., \& Nieuwenstein, M. R. (2017). Cognitive control in media multitaskers: Two replication studies and a meta-analysis. Attention, Perception, and Psychophysics, 79(8), 2620-2641. https://doi.org/10.3758/s13414017-1408-4

Xu, S., Wang, Z., \& David, P. (2016). Media multitasking and well-being of university students. Computers in Human Behavior, 55, 242-250. https://doi.org/10.1016/j.chb.2015.08.040 
Zhao, J., Liu, J., Jiang, X., Zhou, G., Chen, G., Ding, X. P., Fu, G., \& Lee, K. (2016). Linking resting-state networks in the prefrontal cortex to executive function: A functional near infrared spectroscopy study. Frontiers in Neuroscience, 10, Article 452. https://doi.org/10.3389/fnins.2016.00452

\section{Correspondence to:}

Jiutong Luo

Center for Educational Science and Technology, Beijing Normal University at Zhuhai No.18 Jinfeng Road

Zhuhai, 519087

China

Email: jtluo(at)bnu.edu.cn

\&

Chunqi Chang

School of Biomedical Engineering, Shenzhen University

No. 1088, Xueyuan Road

Shenzhen, 518071

China

Email: cqchang(at)szu.edu.cn

Editorial record: First submission received on August 5, 2020. Revisions received on November 21, 2020 and February 10, 2021. Accepted for publication on February 15, 2021.

Editor in charge: Lenka Dedkova

\section{About Authors}

Jiutong Luo is Postdoctoral Research Fellowship at the Advanced Innovation Center for Future Education, Faculty of Education, Beijing Normal University, Beijing, China, and Center for Educational Science and Technology, Beijing Normal University at Zhuhai, Guangdong Zhuhai, China. He received his PhD in Education from the University of Hong Kong. His research interests include educational technology and psychology, children and youth studies, and developmental cognitive neuroscience.

Hui Li is Professor in Early Childhood at Macquarie University, Australia. He received his PhD in Education from the University of Hong Kong. His research interests include (but not limited to) developmental cognitive neuroscience, developmental psycholinguistics, early literacy, curriculum and pedagogy, policy, school leadership, and teacher education. His research methods include mixed-methods, longitudinal study, corpus, and fNIRS.

Pui-sze Yeung is Associate Professor at the Faculty of Education, The University of Hong Kong (HKU). Her research focuses on the links between cognitive-linguistic skills and reading and writing development among Chinese children with and without developmental dyslexia. Her research also examines reading and writing motivation in Chinese children and adolescents.

Chunqi Chang is Professor of Biomedical Engineering at Shenzhen University, China. He was awarded PhD degree by the University of Hong Kong. His research is in the broad areas of signal processing, biomedical engineering, computational systems biology, and brain and cognitive sciences, and his main research interests include neuroimaging, neuroinformatics, neuroengineering, and machine learning. 